\title{
Application of Semi-analytical Finite Plate Strip Method in the Stability Analysis of Rectangular Aqueduct
}

\author{
Yang Zhichao \\ Department of Construction Management \\ Yellow River Henan Bereau \\ ZhengZhou, China \\ yuluem@sina.com
}

Zhang Xiaorui

Project Construction Center

Yellow River Henan Bereau

ZhengZhou, China

11665580@qq.com

\begin{abstract}
The key issue of structural design are three aspects of strength, stiffness and stability. Prestressed rectangular aqueduct that is one of the important hydraulic conveyance also has the same, and it has the "thin" characteristics, and belongs to a slender member, then the stability problem should be paid more attention in the design, Semi-analytical Finite Plate Strip Method solve the problem of the traditional finite element method in the calculation , it is adopted in the local stability analysis of rectangular aqueduct, and it calculates the maximum compressive stress in thin-walled rectangular aqueduct is smaller than the critical instability stress, then rectangular aqueduct can not have local instability, thus it provide a theoritical basis for the stability analysis of rectangular aquduct.
\end{abstract}

Keywords- Aqueduct; Rectangular; Local stability; Semianalytical Finite Plate Strip Method;Thin

\section{INTRODUCTION}

The geometric shape of aqueduct is difficult to describe due to too many freedom degrees, and it is one of the important hydraulic conveyance. In the general, finite element method there are more complicated process and poor precision, While Semi-analytical Finite Plate Strip Method can solve the above problems, then it can simplify calculation, and provides an explicit and simple method. by formula deduction it can establish the Elastic stiffness matrix, geometric matrix of stability of Finite Plate Strip Method[1], and it provides the characteristic equation of structural stability.

\section{FINITE PLATE STRIP ELEMENT METHOD IN STABILITY}

The unit figure of semi-analytical finite strip method is shown as Fig .1. Strain-displacement relationship of large deflection and semi-analytical displacement model[2] are adopt at the same time, i.e , in the calculation discrete Hermit polynomial function is used in the edge,

\author{
$\mathrm{Xu}$ Jinjin \\ School of Civil and Communication \\ North China University of Water Resources and \\ Electric Power \\ ZhengZhou, China \\ 614323440@qq.com
}

Liu Jianzhao

Department of Operation and Observation Yellow River HuiJin Bereau

ZhengZhou, China

672355351@qq.com

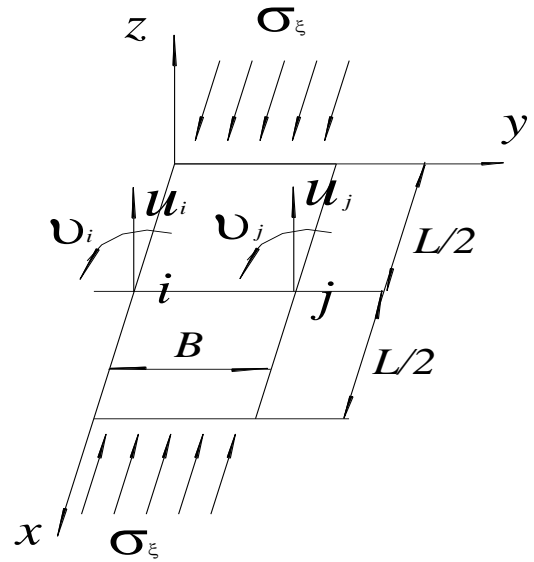

Figure.1 Finite Plate Element Diagram

$x$ direction is consistent with direction of axial compressive load, and the displacement $w$ is a sinusoidal function, the length of the strip element is equal to half instability wavelength, so the normal displacement function can be expressed as

$\boldsymbol{w}=[\psi][\delta] \sin \pi \mathbf{x}$

here

$$
[\psi]=\left[\begin{array}{llll}
\psi_{1} & \psi_{2} & \psi_{3} & \psi_{4}
\end{array}\right]
$$

shape function:

$$
[\psi][\delta]=[\boldsymbol{H}][\mathbf{A}][\delta]
$$

Here

$$
[H]=\left[\begin{array}{llll}
1 & \eta & \eta^{2} & \eta^{3}
\end{array}\right]
$$




$$
\begin{gathered}
{[A]=\left[\begin{array}{cccc}
\frac{1}{2} & \frac{B}{8} & \frac{1}{2} & -\frac{B}{8} \\
-\frac{3}{4} & -\frac{B}{8} & \frac{3}{4} & -\frac{B}{8} \\
0 & -\frac{B}{8} & 0 & \frac{B}{8} \\
\frac{1}{4} & \frac{B}{8} & -\frac{1}{4} & \frac{B}{8}
\end{array}\right]} \\
{[\psi]=[\boldsymbol{H}][\boldsymbol{A}]=\left[\begin{array}{llll}
\psi_{1} & \psi_{2} & \psi_{3} & \psi_{4}
\end{array}\right]}
\end{gathered}
$$

then

$$
\begin{aligned}
& \psi_{1}=\frac{1}{2}-\frac{3}{4} \eta+\frac{1}{4} \eta^{3} \\
& \psi_{2}=\boldsymbol{B}\left(\frac{1}{8}-\frac{1}{8} \eta-\frac{1}{8} \eta^{2}+\frac{1}{8} \eta^{3}\right) \\
& \psi_{3}=\frac{1}{2}+\frac{3}{4} \eta-\frac{1}{4} \eta^{3} \\
& \psi_{4}=\boldsymbol{B}\left(-\frac{1}{8}-\frac{1}{8} \eta+\frac{1}{8} \eta^{2}+\frac{1}{8} \eta^{3}\right)
\end{aligned}
$$

and $\eta=\frac{y}{B}, \eta \in[-1,1], B$ is the width of the plate strip element, $\delta$ is displacement function.

It is easy to conclude this is three Hermite polynomial interpolation[3] during $[0,1]$ between two points.

\section{A Stiffness matrix of finite plate strip element}

\section{1) Geometric Condition}

The geometric equation of thin plate adopt the relationship of stress and strain of Large deflection, it is expressed as:

$$
\begin{aligned}
\{\varepsilon\}=\left\{\begin{array}{l}
-z \frac{\partial^{2} w}{\partial \alpha^{2}}+\frac{1}{2}\left(\frac{\partial w}{\partial \alpha}\right)^{2} \\
-z \frac{\partial^{2} w}{\partial \beta^{2}}+\frac{1}{2}\left(\frac{\partial w}{\partial \beta}\right)^{2} \\
-2 z \frac{\partial^{2} w}{\partial \alpha \partial \beta}+\frac{\partial w}{\partial \alpha} \frac{\partial w}{\partial \beta}
\end{array}\right\} \\
=\left\{\varepsilon^{L}\right\}+\left\{\varepsilon^{N}\right\}
\end{aligned}
$$

Here the $\left\{\varepsilon^{L}\right\}$ is linear strain, and $\left\{\varepsilon^{N}\right\}$ is the nonlinear strain caused by Large deflection.

2) Physical Condition

If the control stress is less than the Proportional limit, then the physical relationship is expressed as:

$$
\{\sigma\}=\left\{\begin{array}{c}
\sigma_{\mathrm{x}} \\
\sigma_{\eta} \\
\tau_{\mathrm{x \eta}}
\end{array}\right\}=\frac{E}{1-\mu^{2}}\left[\begin{array}{ccc}
1 & \mu & 0 \\
\mu & 1 & 0 \\
0 & 0 & \frac{1-\mu}{2}
\end{array}\right]\left\{\begin{array}{c}
\varepsilon_{\mathrm{x}} \\
\varepsilon_{\eta} \\
\gamma_{\mathrm{x \eta}}
\end{array}\right\}
$$

3) The elastic strain energy formula of element

Deformation energy of plate element is expressed as

$U=\frac{1}{2} \int_{v}\{\varepsilon\}^{T}\{\sigma\} \mathrm{d} v=U^{L}+U^{N}$
Here $U$ is the Strain energy of element, $V$ is the volume of integral, $U^{L}$ is Linear strain energy of element, and $U^{N}$ is nonlinear strain energy of element.

4) The Elastic stiffness matrix of element

Elastic stiffness matrix can be deduced by geometric and physical conditions expressed as

$$
\left[K_{E}\right]=\left[K_{E 1}\right]+\left[K_{E 2}\right]+\left[K_{E 3}\right]
$$

Here

$$
\begin{aligned}
& {\left[K_{E 1}\right]=\frac{\pi^{4} E t^{3} B}{10080\left(1-\mu^{2}\right) L^{3}}\left[\begin{array}{cccc}
156 & 22 B & 54 & -13 B \\
22 B & 4 B^{2} & 13 B & -3 B^{2} \\
54 & 13 B & 156 & -22 B \\
-13 B & -3 B^{2} & -22 B & 4 B^{2}
\end{array}\right]} \\
& {\left[K_{E 2}\right]=\frac{\pi^{2} E t^{3}}{36 O\left(1-\mu^{2}\right) B L}}
\end{aligned}
$$$$
\left[\begin{array}{cccc}
36 & C & -36 & 3 B \\
C & 4 B^{2} & -3 B & -B^{2} \\
-36 & -3 B & 36 & -C \\
3 B & -B^{2} & -C & 4 B^{2}
\end{array}\right]
$$

Here

$$
\left[\begin{array}{c}
C=3(1+5 \mu) B \\
{\left[K_{E 3}\right]=\frac{E t^{3} L}{24(1+\mu) B^{3}}\left[\begin{array}{cccc}
12 & 6 B & -12 & 6 B \\
6 B & 4 B^{2} & -6 B & 2 B^{2} \\
-12 & -6 B & 12 & -6 B \\
& 2 B^{2} & -6 B & 4 B^{2}
\end{array}\right]}
\end{array}\right.
$$

5) Geometric stiffness matrix of element

Considering the nonlinear strain energy the geometric stiffness matrix can be expressed as

$$
\left[K_{G}\right]=\left\lfloor K_{G \xi}\right\rfloor+\left\lfloor K_{G \eta}\right\rfloor+\left\lfloor K_{G \xi \eta}\right\rfloor
$$

Here $\left\lfloor K_{G \xi}\right\rfloor$ is the condition of longitudinal pressure, $\left\lfloor K_{G \eta}\right\rfloor$ is the condition of Lateral pressure, and $\left\lfloor K_{G \xi \eta}\right\rfloor$ is the condition of pure torsion. the stability od aqueduct only has the $\left[K_{\mathrm{G} \eta}\right]$, then the Geometric stiffness matrix of element is expressed as:

$$
\left[K_{G}\right]=\frac{\sigma_{\eta} \pi^{2} B t}{840 L}\left[\begin{array}{cccc}
156 & 22 B & 54 & -13 B \\
22 B & 4 B^{2} & 13 B & -3 B^{2} \\
54 & 13 B & 156 & -22 B \\
-13 B & -3 B^{2} & -22 B & 4 B^{2}
\end{array}\right]
$$

and these are the general form of plate element.

5) contrl stress of stability

Elastic stiffness matrix and geometric stiffness matrix of structure can get by stiffness integration method, then the stiffness equation can get, and solve the maximum 
characteristic root of characteristic equation $\left(1 / q_{c r}\right)_{\max }$,and its reciprocal is exactly the minimum critical instability load that we require[4]. Then,we can get the vector $\{\delta\}$ describing structural configuration of instability after getting critical load.

\section{SOLUTION OF THE MAXIMUM COMPRESSIVE STRESS IN RECTANGULAR AQUEDUCT}

The cross section dimension of rectangular aqueduct is shown in figure 2. y1 is the distance section centroid to the half upper axis, y2 is the section centroid to the lower axis[5][6].

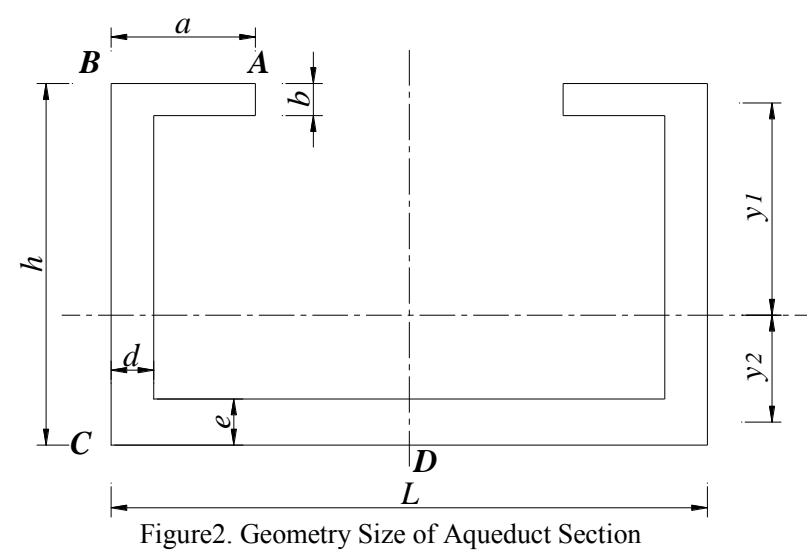

\section{B The Calculation of the Normal Stress}

The calculation of normal stress in cross-section of aqueduct adopt the same equation as bending cross-section which is applied lateral force, the formula is as follows

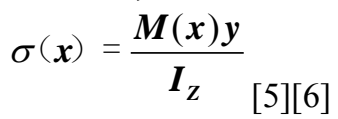

\section{C .The Calculation of Shear Stress}

The distribution law of shearing stress in cross-section of rectangular beams[5] [6] can be concluded that its distribution along the centerline of the cross section and which is neglected in the direction of thickness, because there is big difference in size of the thickness and section, thus the shearing stress formula in each section is as follows

$$
\begin{gathered}
\tau_{A B}=\frac{\boldsymbol{F}_{s}(\boldsymbol{x}) \boldsymbol{y}_{1} \boldsymbol{s}}{\boldsymbol{I}_{z}} \quad \mathbf{0} \leq \mathbf{s} \leq \boldsymbol{a}-\frac{\boldsymbol{d}}{2} \\
\tau_{B C}=\frac{\boldsymbol{F}_{S}(\boldsymbol{x})\left[\boldsymbol{a b} y_{1}+\boldsymbol{d}(\boldsymbol{s}-\boldsymbol{b})\left(\boldsymbol{y}_{1}-\frac{\boldsymbol{s}}{2}\right)\right]}{\boldsymbol{I}_{z} \boldsymbol{d}} \\
\mathbf{0} \leq \mathbf{s} \leq \boldsymbol{y}_{1}+\boldsymbol{y}_{2}
\end{gathered}
$$

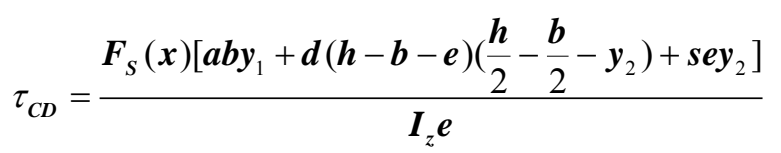

$$
\mathbf{0} \leq \mathbf{s} \leq \frac{\boldsymbol{L}}{2}-\frac{\boldsymbol{d}}{2}
$$

where $\tau_{A B}, \tau_{B C}, \tau_{C D}$ are used respectively to express shearing stress in sections of $A B, B C, C D, s$ is the curvilinear coordinate in the direction of shear stress stream , $I_{Z}$ is the inertia moment of cross section to central axis.

\section{Calculation of the Maximum Compressive Stress}

Stresses in the same point but different section have different magnitudes according to strength theory, then if the maximum stress that is principal stress will be larger than the maximum stress on cross section of beam? Then calculate the maximum stress on section by solve the derivation of function of principal stress, the principal stress formula calculating magnitude and direction is as follows:

$$
\begin{gathered}
\sigma_{c \max }=\frac{\sigma}{2}-\sqrt{\left(\frac{\sigma}{2}\right)^{2}+\tau_{x}^{2}} \\
\alpha=\frac{1}{2} \arctan \left(\frac{-2 \tau_{x}}{\sigma}\right)
\end{gathered}
$$

Where $\sigma_{c \max }$ is the maximum compressive stress, $\alpha$ is the angle between the maximum compressive stress and $\mathrm{x}$ axis, and it would be positive when it is counter-clockwise.

On different sections $\sigma_{c \max }$ is a function about $\mathrm{y}$, then working out the magnitude and direction of the maximum compressive stress in the point by calculating $\frac{d \sigma_{c \max }}{\boldsymbol{d y}}=0$

[8], finally we can get the maximum compressive stress of aqueduct in normal operation.

\section{ENGINEERING APPLICATION}

The project of South-to-North Water Transfer crossing yellow river adopts rectangular aqueduct with thin wall, the flow of water is $120 \mathrm{~m} 3 / \mathrm{s}$, basic dimensions of section are as follows: $\mathrm{a}=1 \mathrm{~m}, \mathrm{~b}=0.2 \mathrm{~m}, \mathrm{~h}=5.4 \mathrm{~m}, \mathrm{~d}=0.5 \mathrm{~m}, \mathrm{~L}=12 \mathrm{~m}$, $\mathrm{e}=0.5 \mathrm{~m}$, considering the symmetry of aqueduct ,calculation should be within the span of $l / 2$, there are 48 elements in calculation, the top flange has 3 elements, side wall has 25 elements, and there are 18 elements above neutral axis, the other 7 elements under neutral axis, the bottom has 20 elements, there are 49 nodes, and there is $w_{4}=w_{29}=w_{49}=0$ by symmetric instability boundary condition, in the antisymmetric instability boundary condition there is $w_{4}=w_{29}=\theta_{49}=0$ [9]. The element size in the corner parts is properly reduced to ensure the accurate of the calculation results, the maximum principal stress is as table 1 shows:

Table I.Value of the Maximum Principal Compressive Stress Indifferent Sections $(\mathrm{MPa})$

\begin{tabular}{ccccc}
\hline $\begin{array}{l}\text { position } \\
\text { of section }\end{array}$ & $1 / 2$ & $1 / 4$ & $1 / 8$ & $1 / 16$ \\
\hline $\begin{array}{c}\sigma_{c \max } \\
\left(q l^{2}\right)\end{array}$ & 0.014934 & 0.011201 & 0.010284 & 0.010936 \\
\hline
\end{tabular}


Where $q, l$ is the upper load applied on aqueduct and the span of aqueduct respectively, international system of units is adopt in the process of calculating, the unit of

$\sigma_{c \max }$ is $\mathrm{MPa}$, the maximum compressive stress15.54MPa in top flange plate and siding shingle locates on the top flange plate of mid-span, while the maximum stress of top flange plate and siding shingle is $32 \mathrm{MPa}$ calculated by Semi-analytical Finite Plate Strip Method ( considering the critical stress that is got in the condition of elastic instability is greater than strength limit of C50 concrete[10] and the former can not be larger than the latter, so instead the former with the latter). If the structure occurs failure it may be plastic instability or overload of pressure[11][12]. Instability wavelength is $6.3 \mathrm{~m}$. In conclusion, the instability critical stress on aqueduct structure is larger than the maximum compressive stress that aqueduct body can bear, then the flange, siding shingle and groove bottom of aqueduct structure can not be in local instability failure[13][14].

\section{CONCLUSIONS}

It is resulted that the maximum compressive stress of rectangular aqueduct occurs on the upper flange of crosssection of mid-span by calculation and analysis, as the critical instability stress of structure calculated by Semianalytical Finite Plate Strip Method is larger than the maximum compressive stress that aqueduct can bear, so we can check the stability of structure by maximum compressive stress of aqueduct..

Semi-analytical Finite Plate Strip Method can ensure the geometric shape coincide with actual structure in discreting, it has advantages in describing instability configuration easily and less degrees of freedom, in a short, it is a simple, practical and precise manner of calculation. Simply supported rectangular thin-waller beam aqueduct has great reserve of stability safety, so it will not be in failure of stability.

\section{REFERENCES}

[1] Liu Dongchang, Liu Xianliang, Meng Wenyuan. Finite element method of stability analysis of prestressed u-shaped thin shell slot structure. Journal of HeHai university, 1996,(12):86-90

[2] Liu Dongchang, Liu Xianliang, Ji Dong. Study of the problem of overall stability of $\mathrm{u}$-shaped thin shell aqueduct. The Yellow River planing and design, 1996 (12):39-43

[3] Zhang DuoXin, Liu Dongchang, Jia Zhiying. The analysis and calculation of prestressed $\mathrm{u}$-shaped thin shell stability[J], tunnel construction, 2003, 23 (6):10-11

[4] The Ministry of Water Resources of The People's Republic of China, Design code for hydraulic concrete structures (SL/T19196) ,Beijing, China Water Power Press,In 1997

[5] Xunfang Sun, Xiaoshu Fang, Laitai Guan, Mechanics of Materials (Volume One、 Volume two),Beijing, Higher Education Press, In 2009

[6] Hongwen Liu, Mechanics of Materials (Volume One、 Volume two),Beijing, Higher Education Press, In 2006

[7] Dongchang Liu, Study of finite element method to estimate local buckling stress of stiffened panels in axial compression,Acta Aeronautica et Astroautica Sinica,In 1981,(3)

[8] Zhengsen Song, Qimei Zhang. Flume design and computer program [M],Jinan,Press of science and technology of Shandong province,In 1997,(7)

[9] Alan Burrows. Wastewater - Aqueduct project uses vertical power [J]. World Pumps, 2012, (TN.550):30-31.

[10] Delfin, E.L.,Gaushell, D.J. Integration of computer systems for California aqueduct power plant systems [J]. IEEE Transactions on Energy Conversion,1993,8(2):190-196.

[11] Yuchun Li,Qingshuang Di,Yongqing Gong et al. Equivalent mechanical models of sloshing fluid in arbitrary-section aqueducts [J]. Earthquake engineering and structural dynamics, 2012, 41(6):1069-1087.

[12] S. Benjamin Valdez,W. Michael Schorr,G. Navor Rosas et al Corrosion Control in the Rio Colorado-Tijuana Aqueduct [J]. Corrosion reviews, 2005,23(4/5/6):247-276.

[13] Hua Zhang,Liang Liu,Ming Dong et al. Analysis of wind-induced vibration of fluid-structure interaction system for isolated aqueduct bridge [J]. Engineering structures,2013,46(Jan.):28-37.

[14] Benjamin Valdez,Navor Rosas,Jose A. Sampedro et al.Corrosion of reinforced concrete of the Rio Colorado-Tijuana Aqueduct [J] Materials Performance,1999,38(5):80-82. 